Pacific

Journal of

Mathematics

\title{
GENERALIZED NUCLEI OF COMPLEX SURFACES
}

\author{
TERRY FulLer
}

Volume $187 \quad$ No. 2

February 1999 


\title{
GENERALIZED NUCLEI OF COMPLEX SURFACES
}

\author{
TERRY FULLER
}

\begin{abstract}
For each $g \geq 1$, we study a family $Y_{g}(n)$ of complex surfaces which admit a singular fibration over $\mathbb{C} P^{1}$ by complex curves of genus $g$. By examining a handlebody description for $Y_{g}(n)$, we show that these complex surfaces can be smoothly decomposed as the Milnor fiber of a Brieskorn homology 3-sphere union a small submanifold, termed a "nucleus". This description generalizes known decompositions for elliptic surfaces.
\end{abstract}

\section{Introduction.}

Elliptic surfaces have long been an important source of examples in the study of smooth 4-manifolds. One important step in their study was the discovery by Gompf of "nuclei" of elliptic surfaces, that is, of small submanifolds which carry much of the differential topological information of the larger elliptic surface $[\mathbf{G}]$. Furthermore, Gompf identified the complements of nuclei as familiar examples (a certain family of Milnor fibers of Brieskorn homology 3-spheres), yielding a decomposition for elliptic surfaces that has proven useful in a variety of contexts. (See $[\mathbf{F S}],[\mathbf{G}],[\mathbf{G M}],[\mathbf{L M}]$ for applications.)

The situation for higher genus fibrations, however, is less settled. In this paper, for each $g \geq 1$ we study a family $Y_{g}(n)$ of complex surfaces admitting a singular fibration over $\mathbb{C} P^{1}$ by genus $g$ Riemann surfaces, describing their topology by means of Kirby calculus. These complex surfaces are shown to have a decomposition analogous to Gompf's decomposition for elliptic surfaces: each $Y_{g}(n)$ contains a small $\left(b_{2}=2\right)$ submanifold, also referred to as a nucleus, whose complement is diffeomorphic to the Milnor fiber of the Brieskorn homology 3 -sphere $\Sigma(2,2 g+1,2(2 g+1) n-1)$. Indeed, for $g=1$, we recover precisely the decomposition of $[\mathbf{G}]$ for elliptic surfaces.

\section{A Family of Branched Covers.}

For all $k \geq 0$, let $\mathbb{F}_{k}$ denote the $k$ th Hirzebruch surface, the holomorphic $\mathbb{C} P^{1}$-bundle over $\mathbb{C} P^{1}$ with a holomorphic section of self-intersection number $-k$. As a smooth 4-manifold, we will find it convenient to think of $\mathbb{F}_{k}$ as the double of a $D^{2}$-bundle over $S^{2}$ with Euler number $k$, and hence $\mathbb{F}_{k}$ can be described by the handlebody in Figure $1 . \mathbb{F}_{k}$ admits two disjoint holomorphic sections $\Delta_{k}$ and $\Delta_{-k}$, with $\Delta_{ \pm k}^{2}= \pm k$. 
For all $g \geq 1$ and $n \geq 1$, we define $Y_{g}(n)$ to be the 2-fold cover of $\mathbb{F}_{2 n}$, branched over the disjoint union of a smooth curve in $\left|(2 g+1) \Delta_{2 n}\right|$ and $\Delta_{-2 n}$. $Y_{g}(n)$ admits a singular fibration $Y_{g}(n) \rightarrow \mathbb{C} P^{1}$ obtained by composing the branched cover map with the bundle map $\mathbb{F}_{2 n} \rightarrow \mathbb{C} P^{1}$. Since a typical nonsingular fiber appears as the double cover of a sphere branched over $2 g+2$ points, this is a singular fibration by genus $g$ curves. This fibration admits a holomorphic section $S$ with $S^{2}=-n$, obtained as the lift of $\Delta_{-2 n}$ to $Y_{g}(n)$.

\section{A handlebody description of $Y_{g}(n)$.}

Our results follow by obtaining a handlebody description for $Y_{g}(n)$, which is used to understand the structure of the singular fibration $Y_{g}(n) \rightarrow \mathbb{C} P^{1}$. Because of the large Euler characteristics involved, we discuss the development of handlebody pictures for the family $Y_{2}(n)$ of genus 2 fibrations in detail, and comment later on the obvious modifications for $Y_{g}(n)$. This handlebody description is also discussed in $[\mathbf{F u}]$ for the special case $Y_{2}(1)$.

Special Case: $Y_{2}(n)$. Setting $g=2$, we begin by constructing a smooth complex curve representing $\left[5 \Delta_{2 n}\right] \in H_{2}\left(\mathbb{F}_{2 n}\right)$. To do this, we construct the $D^{2}$-bundle over $S^{2}$ with Euler number $2 n$ by gluing two copies of $\mathbb{C}^{2}$ with coordinates $(w, \eta)$ and $(z, \xi)$ according to the identifications

$$
\begin{aligned}
& w=z^{-1} \\
& \eta=z^{-2 n} \xi .
\end{aligned}
$$

We can describe five copies of $\Delta_{2 n}$ in the $(w, \eta)$ chart as the set of all points $\left(w, \eta_{1}\right), \ldots,\left(w, \eta_{5}\right)$, where $\eta_{1}, \ldots, \eta_{5}$ are the five roots of unity, and extend these over the $(z, \xi)$ chart by setting $\xi_{i}=\eta_{i} z^{2 n}$ for $1 \leq i \leq 5$. These five copies intersect at $z=0$. These five copies are thus given locally by the complex curve

$$
\left(\xi-\eta_{1} z^{2 n}\right) \cdots\left(\xi-\eta_{5} z^{2 n}\right)=\xi^{5}-z^{10 n}=0,
$$

and we can smooth out the intersection point by deforming this curve into

$$
\xi^{5}=z^{10 n}+\epsilon .
$$

Figure 2 shows $\mathbb{F}_{2 n}$ along with a piece of the smoothed branch surface, which is known to be given by the fibered Seifert surface for the $(5,10 n)$ torus link ([Mi] $)$. We label the 2-handles of $\mathbb{F}_{2 n}$ as $h$ and $h^{\prime}$, for later reference. The boundary of this surface is five circles; the rest of the surface is five disjoint smoothly embedded disks $D_{1}, \ldots, D_{5}$ in $\partial$ (4-handle), obtained from parallel copies of the core of $h^{\prime}$. The other component of the branch set is $\Delta_{-2 n}$, which is seen in Figure 2 as the cocore of $h$ union an unseen disk $D_{6}$ in the 4-handle. Sliding $h^{\prime} 2 n$ times over $h$ produces Figure 3. The images of the disks $D_{1}, \ldots, D_{5}$ (which we continue to call $D_{1}, \ldots, D_{5}$ ) under this 
diffeomorphism are parallel copies of the $-2 n$-framed 2-handle (which we continue to call $h^{\prime}$ ). Moreover, since the handle slides give a diffeomorphism from $\mathbb{F}_{2 n}$ to its upside down handlebody description, the disk $D_{6}$ is also seen in Figure 3 as a parallel copy of the core of $h^{\prime}$. Figure 4 shows the result of an isotopy, where the five flat disks in Figure 3 have been placed horizontally across the page. (We leave it to the reader to check that this isotopy untwists the half-twisted bands of Figure 3.)

Figure 5 shows the 2-fold branched cover $Y_{2}(n)$ of $\mathbb{F}_{2 n}$, drawn from Figure 4 using the methods of $[\mathbf{A K}]$. To understand Figure 5, it is best to picture it being built in stages. We begin by picturing the branch surface as the six 0 -handles $\cup 40 n$ 1-handles in $\partial\left(0\right.$-handle) of $\mathbb{F}_{2 n}$ visible in Figure 4 , union the 2-handles $D_{1}, \ldots, D_{6}$ in the 4 -handle of $\mathbb{F}_{2 n}$. To form the branched cover $Y_{2}(n)$, we lift the handles of $\mathbb{F}_{2 n}$ one at a time. To lift the 0-handle of $\mathbb{F}_{2 n}$, we isotop the interior of the six 0-handles $\cup 40 n$ 1-handles into the interior of the 0 -handle of $\mathbb{F}_{2 n}$. We then cut each of two copies of the 0 -handle of $\mathbb{F}_{2 n}$ along the track of this isotopy, and glue the resulting manifolds together by attaching handles. Gluing two copies of the 0-handle of $\mathbb{F}_{2 n}$ along the six 0 -handles of the branch set is accomplished by attaching six 1-handles with each component of the attaching region in a separate copy, or equivalently by attaching five 1-handles to a single 4-ball. These five 1-handles are the dotted circles in Figure 5. Additional gluings along the $40 n$ 1-handles of the branch set are obtained by attaching the $40 n$ 0-framed 2-handles in Figure 5 .

To lift the 2-handles $h$ and $h^{\prime}$, we use the method of [AK], Section 3. The attaching circle of $h^{\prime}$ intersects the branch set geometrically in $2 n$ points. We lift $h^{\prime}$ to two 2-handles $h_{1}^{\prime}$ and $h_{2}^{\prime}$ that are attached to circles obtained by cutting the attaching circle of $h^{\prime}$ along the branch set, and gluing the endpoints together as we perform the gluing of the 0-handles of $\mathbb{F}_{2 n}$. We compute, as in $[\mathbf{A K}]$, that the framings of $h_{1}^{\prime}$ and $h_{2}^{\prime}$ are $-n$. Similarly, $h$ lifts to 2-handles $h_{1}$ and $h_{2}$, each with framing -3 . Lastly, we must lift the 4-handle of $\mathbb{F}_{2 n}$. However, the lift is obtained from two copies of the 4-handle glued along the (pushed in) disks $D_{1}, \ldots, D_{6}$, hence by turning the initial part of our argument upside down it appears as five 3-handles $\cup$ 4-handle.

We next modify this picture of $Y_{g}(n)$. We begin by isotoping the wheels of 0 -framed 2-handles so that the two half twists per wheel are turned into one full twist, resulting in Figure 6. The 1-handles are then isotoped by sliding each dotted circle over the other dotted circles to its right; this rearranges the dotted circles so that each wheel runs through a single dotted circle. Cancelling four of the 1-handles with the outermost 2-handle from each wheel, and cancelling the fifth 1 -handle with one of the -3 -framed 2 -handles produces Figure 7 . Figure 8 is obtained by sliding one of the $-n$-framed 2 handles over the other, splitting off an unknotted 0-framed 2-handle from 
the rest of the picture, which is used to cancel one of the 3-handles. The handlebody in Figure 9 is diffeomorphic to that in Figure 8, as can be seen by cancelling each 1-handle with the extra 2-handle in each wheel (and isotoping the 0 -framed $(2,5)$ torus knot).

From Figure 9, we can extract a description of the singular fibers of $Y_{2}(n) \rightarrow \mathbb{C} P^{1}$. Viewing Figure 9 in stages, attaching first the 1-handles and the 0 -framed 2-handle (see Figure 10) describes $\Sigma_{2} \times D^{2}$, a trivial fibration over $D^{2}$ by genus 2 surfaces. The $40 n-1$-framed 2 -handles are attached next with attaching circles lying in fibers $\Sigma_{2} \times\{p t.\} \subset \Sigma_{2} \times S^{1}=\partial\left(\Sigma_{2} \times D^{2}\right)$. This is known (see $[\mathbf{H K K}],[\mathbf{K}]$ ) to be a handlebody description of a singular fibration over $D^{2}$ with $40 n$ singular fibers, each of which is an immersed genus 2 surface with one transverse self-intersection, obtained from nearby fibers by identifying one of the circles $c_{1}, c_{2}, c_{3}, c_{4}$ (see Figure 11) to a point. The monodromy around each is given by $D\left(c_{i}\right)$, a right-handed Dehn twist around $c_{i}$. The boundary is an $\Sigma_{2}$-bundle over $S^{1}$ whose global monodromy is the product of those Dehn twists. Since a meridianal circle to the 0-framed 2-handle in Figure 9 intersects each fiber in the boundary once, traversing this circle allows us to read off this monodromy as

$$
\left(D\left(c_{1}\right) D\left(c_{2}\right) D\left(c_{3}\right) D\left(c_{4}\right)\right)^{10 n} .
$$

Finally, the submanifold given by the $-n$-framed 2 -handle $\cup$ four 3 -handles $\cup$ 4-handle in Figure 9 is diffeomorphic (by tracing it back to Figure 5) to the 2-fold cover of the submanifold $h^{\prime} \cup 4$-handle branched over the pushed in disks $D_{1}, \ldots, D_{6}$. Since these disks are all parallel copies of $h^{\prime}$, this is diffeomorphic to the 2 -fold cover of a trivial $D^{2}$-bundle over $S^{2}$ branched over six fibers, namely $\Sigma_{2} \times D^{2}$. Hence adding the $-n$-framed 2 -handle $\cup$ four 3-handles $\cup 4$-handle to complete Figure 9 describes a $\Sigma_{2} \times D^{2}$ attached to the boundary, preserving the $\Sigma_{2}$-bundle structure. In particular, the global monodromy above must be trivial. (The word $\left(D\left(c_{1}\right) D\left(c_{2}\right) D\left(c_{3}\right) D\left(c_{4}\right)\right)^{10 n}$ is known to be trivial in the mapping class group of a genus 2 surface $[\mathbf{B}]$.)

Figure 12(a) shows a regular neighborhood $C$ of four consecutive singular fibers, whose singularities are defined by the circles $c_{1}, c_{2}, c_{3}$, and $c_{4}$, respectively. Cancelling the 1-handles produces a 4-manifold obtained by attaching a 2 -handle to $B^{4}$ along a 0 -framed $(2,5)$ torus knot $K$, as in Figure $12(\mathrm{~b})$. With this description, since $K$ is a fibered knot with genus 2 fiber, we have a fibration on $C$ with only one singular fiber, called a cusp fiber. (We have described the genus 2 analog of the familiar statement from elliptic surfaces that two fishtail fibers can be deformed into one cusp fiber.) Examining the global monodromy $\left(D\left(c_{1}\right) D\left(c_{2}\right) D\left(c_{3}\right) D\left(c_{4}\right)\right)^{10 n}$, we see that the original fibration with $40 n$ singular fibers is diffeomorphic to a fibration with $10 n$ cusp fibers.

Following the terminology coined in $[\mathbf{G}]$, we make the following definition. 
Definition 1. The nucleus $N_{2}(n) \subset Y_{2}(n)$ is a regular neighborhood of a single cusp fiber union the section $S$.

Since $S$ intersects each fiber in $C$ once, Figure 13 gives a Kirby calculus picture of $N_{2}(n)$. We next show that the decompositions of elliptic surfaces in $[\mathbf{G}]$ apply here as well.

Theorem 2. The complement of $N_{2}(n)$ in $Y_{2}(n)$ is diffeomorphic to $B(2,5$, $10 n-1)$, the Milnor fiber of the Brieskorn homology 3-sphere $\Sigma(2,5,10 n-1)$.

Proof. The argument in $[\mathbf{G}]$ generalizes intact to our pictures. It follows from the above discussion that drawing Figure 9 without the $-n$-framed 2 handle $\cup$ four 3 -handles $\cup$ 4-handle describes $Y_{g}(n)-\stackrel{\circ}{\nu}(f)$, the complement of a regular neighborhood of a nonsingular fiber $f$. To describe $Y_{g}(n)-$ $\stackrel{\circ}{C}$, we further leave out one -1 -framed 2 -handle per wheel. To get the complement of $N_{g}(n)$, we must additionally delete a neighborhood of $S$ restricted to $Y_{g}(n)-\stackrel{\circ}{C}$. However, the cocore of the 0 -framed 2-handle is a properly embedded disk in $Y_{g}(n)-\stackrel{\circ}{C}$ which extends to $S$ in $Y_{g}(n)$. Hence deleting this disk, or equivalently omitting the 0-framed 2-handle, describes the complement of $N_{g}(n)$ in $Y_{g}(n)$. Figure 14 shows what has survived from Figure 9. Cancelling the 1-handles produces Figure 15.

The Milnor fiber $B(2,5,10 n-1)$ is by definition the solution set of $x^{2}+$ $y^{5}+z^{10 n-1}=\varepsilon$ in the unit ball of $\mathbb{C}^{3}$. This is easily seen (see the discussion in $[\mathbf{A K}])$ to be diffeomorphic to the $(10 n-1)$-fold cover of $B^{4}$, branched over the fibered Seifert surface for a $(2,5)$ torus knot whose interior has been pushed into $B^{4}$. Applying the methods of $[\mathbf{A K}]$ to this branched cover also yields Figure 15 .

The General Case: $Y_{g}(n)$. We can easily extend this discussion to describe the analogous pictures for arbitrary $g$. To depict a smooth complex curve in $\left|(2 g+1) \Delta_{2 n}\right|$, we replace the Seifert surface of a $(5,10)$ torus link in Figure 2 with the Seifert surface for a $(2 g+1,2(2 g+1) n)$ torus link, drawn as $2 g+1$ disks connected by $4 g n(2 g+1)$ twisted bands. Carrying through the same analysis as before produces the picture of $Y_{g}(n)$ in Figure 16. In this case, we consider a regular neighborhood $C$ of $2 g$ consecutive singular fibers whose monodromies are given by Dehn twists around the circles $c_{1}, \ldots, c_{2 g}$ (see Figure 17). $C$ can be obtained by attaching a 2-handle to $B^{4}$ along a 0 -framed $(2,2 g+1)$ torus knot, which is taken to be the genus $g$ analog of the neighborhood of a cusp fiber. As before, if we delete the $-n$-framed 2-handle $\cup 2 g$ 3-handles $\cup$ 4-handle from Figure 16, then the boundary is a $\Sigma_{g}$-bundle over $S^{1}$ with global monodromy

$$
\left(D\left(c_{1}\right) D\left(c_{2}\right) \cdots D\left(c_{2 g}\right)\right)^{2(2 g+1) n},
$$


and we see that $Y_{g}(n)$ is diffeomorphic to the total space of a fibration with $2(2 g+1) n$ cusp fibers. Once again, we define the nucleus $N_{g}(n) \subset Y_{g}(n)$ to be a regular neighborhood of a cusp fiber union the section $S$, so that $N_{g}(n)$ appears as in Figure 18. With these definitions, the following theorem follows as before.

Theorem 3. The complement of $N_{g}(n)$ in $Y_{g}(n)$ is diffeomorphic to $B(2$, $2 g+1,2(2 g+1) n-1)$, the Milnor fiber of the Brieskorn homology 3-sphere $\Sigma(2,2 g+1,2(2 g+1) n-1)$.

Since the Milnor fibers $B(2,2 g+1,2(2 g+1) n-1)$ have an even intersection form, we have also shown the following corollary.

Corollary 4. $Y_{g}(n)$ is spin if an only if $N_{g}(n)$ is spin.

Indeed, this fact is apparent from comparing Figures 16 and 18. In fact, from these Figures we see that $Y_{g}(n)$ and $N_{g}(n)$ are spin precisely when $n$ is even.

In addition, examining Figure 16 more carefully, we note as before that the - $n$-framed 2-handle $\cup 2 g$ 3-handles $\cup$ 4-handle represent a $\Sigma_{g} \times D^{2}$ attached to the boundary of its complement, preserving the $\Sigma_{g}$-bundle structure. Thus the global monodromy recorded above must be trivial. In particular, setting $n=1$, this gives a geometric explanation for the following well-known fact $[\mathbf{B}]$.

Corollary 5. The product of Dehn twists $\left(D\left(c_{1}\right) D\left(c_{2}\right) \cdots D\left(c_{2 g}\right)\right)^{2(2 g+1)}$ represents the trivial element in the mapping class group of $\Sigma_{g}$. 


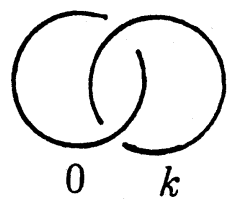

$\cup$-h

Figure 1.

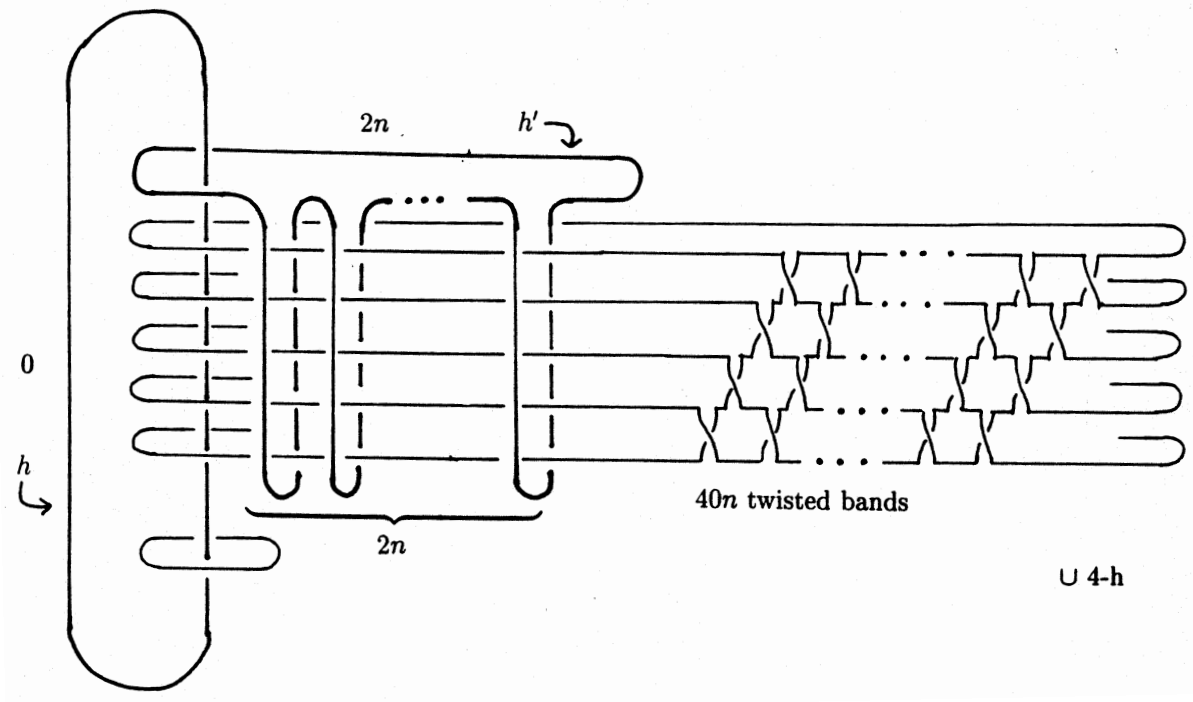

Figure 2. 


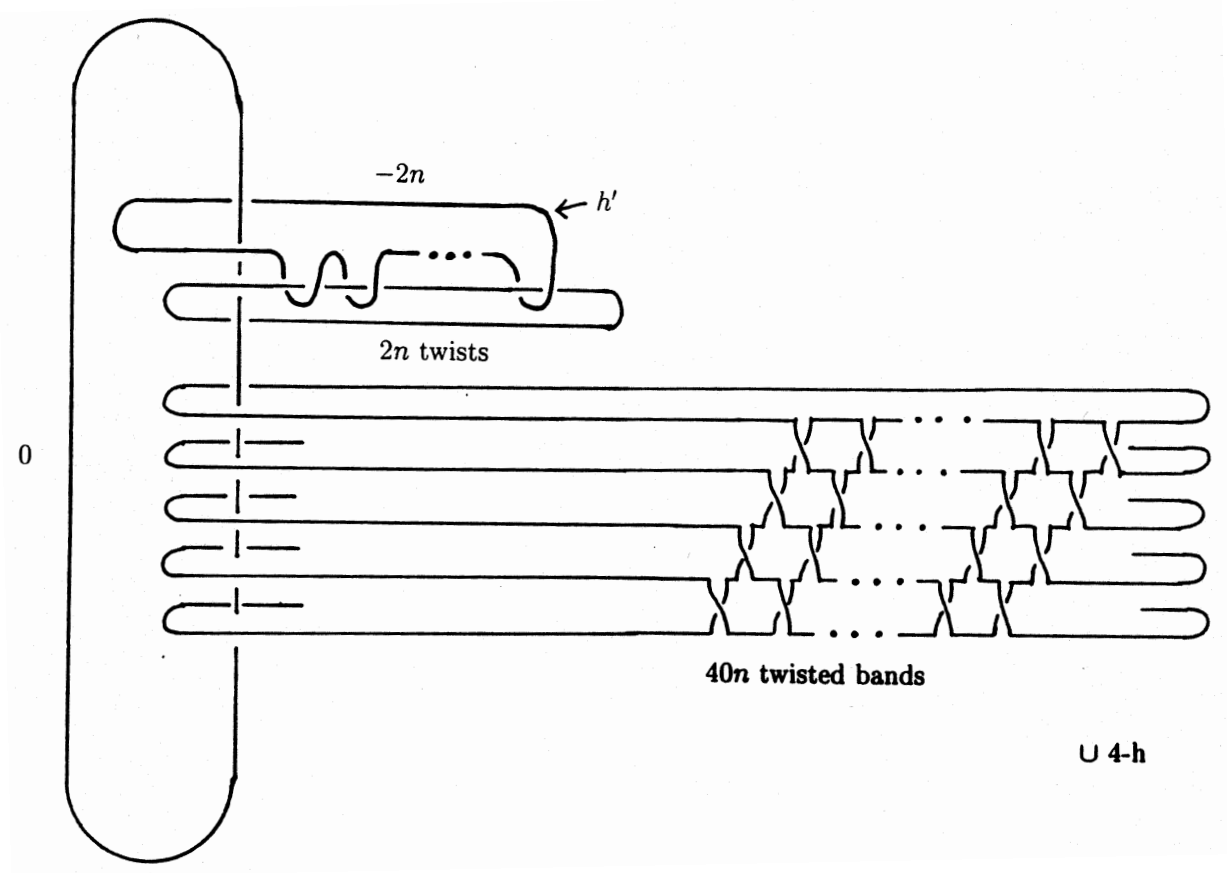

Figure 3.

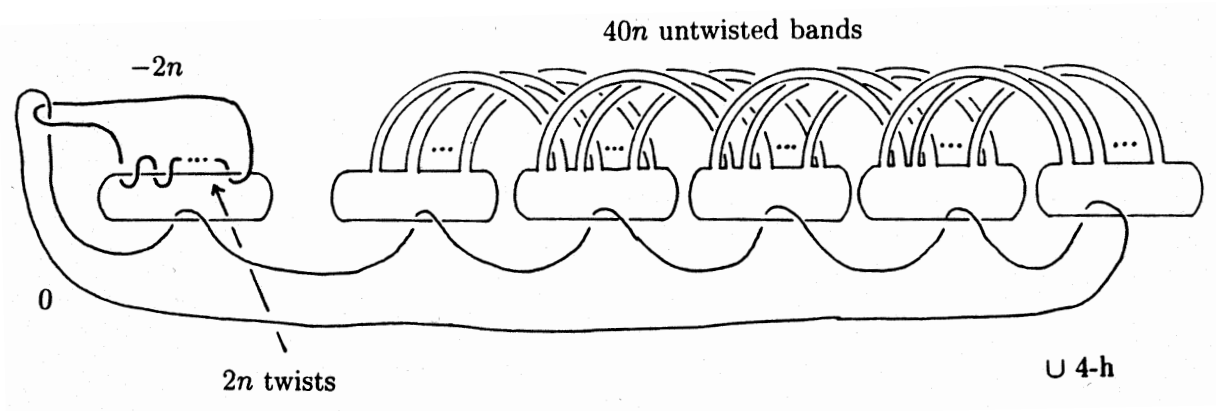

Figure 4. 


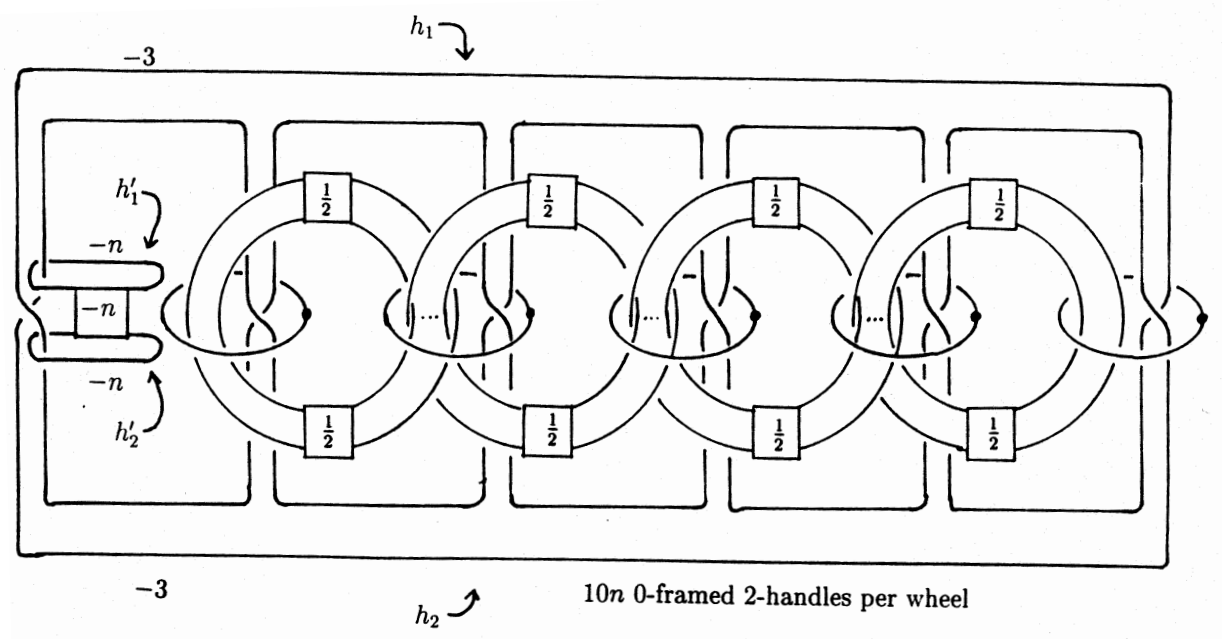

U five 3-h, 4-h

Figure 5.

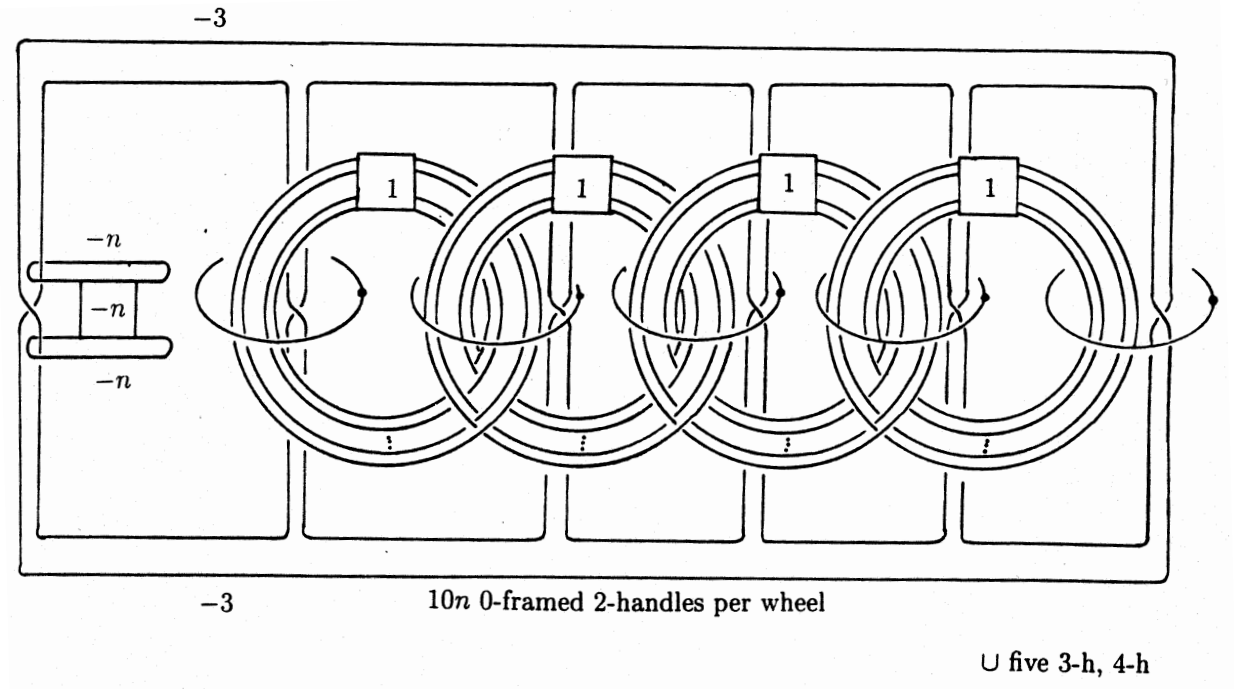

Figure 6. 


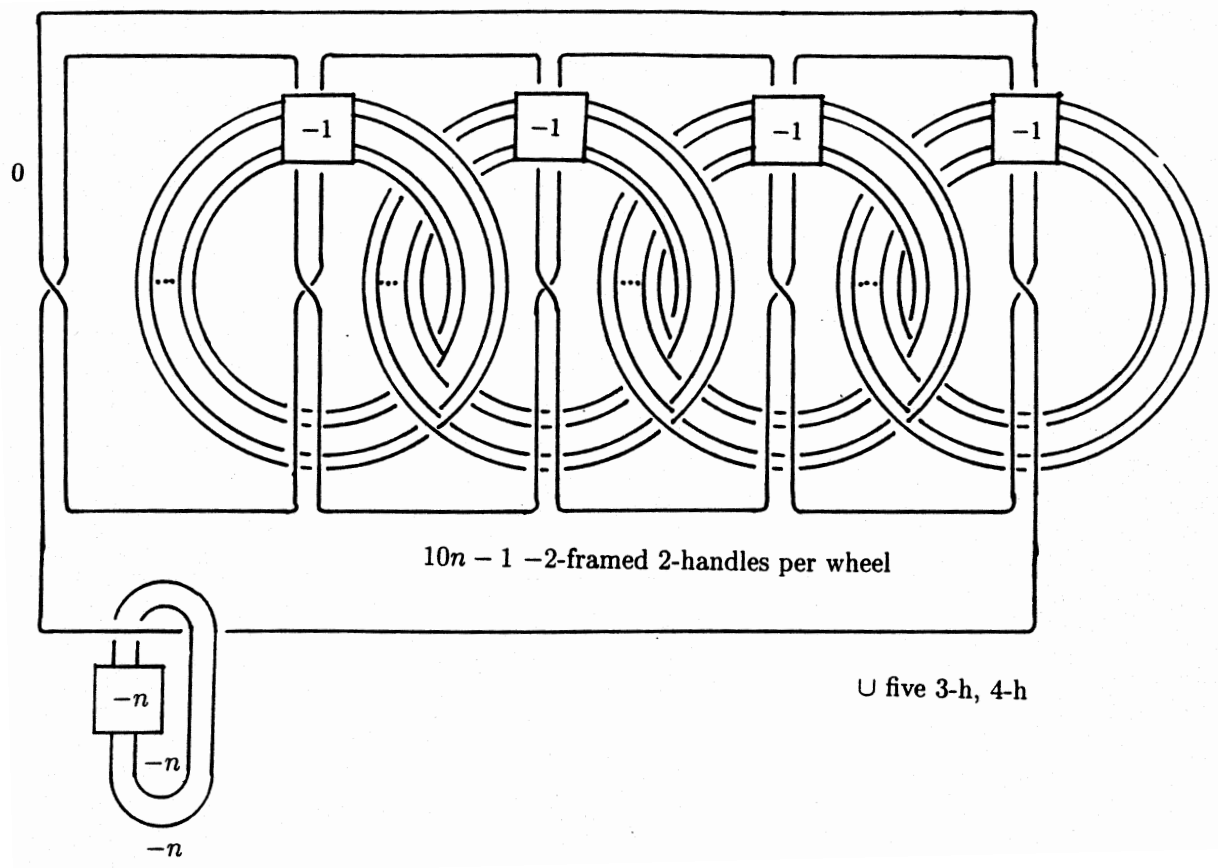

Figure 7.

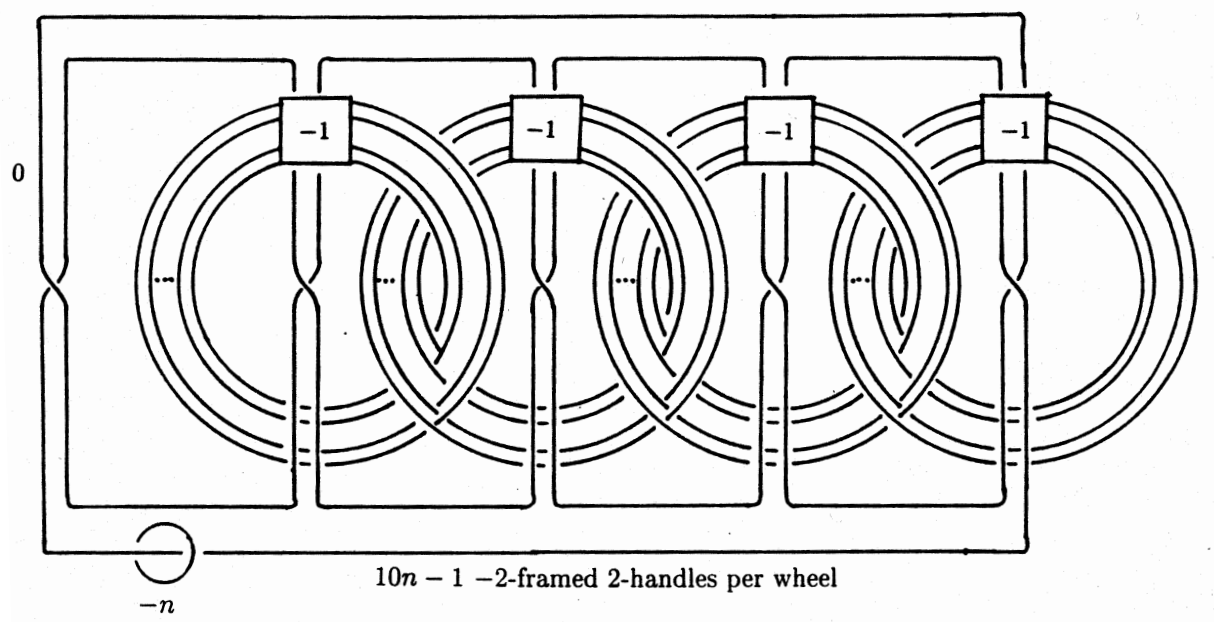

$U$ four 3-h, 4-h

Figure 8. 


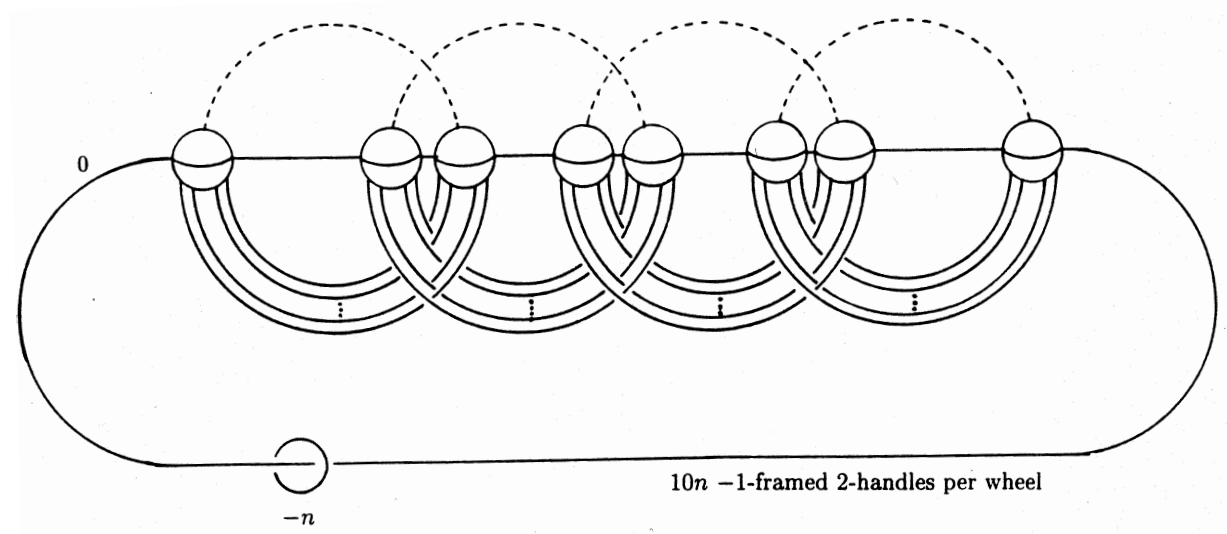

$\cup$ four 3-h, 4-h

Figure 9.

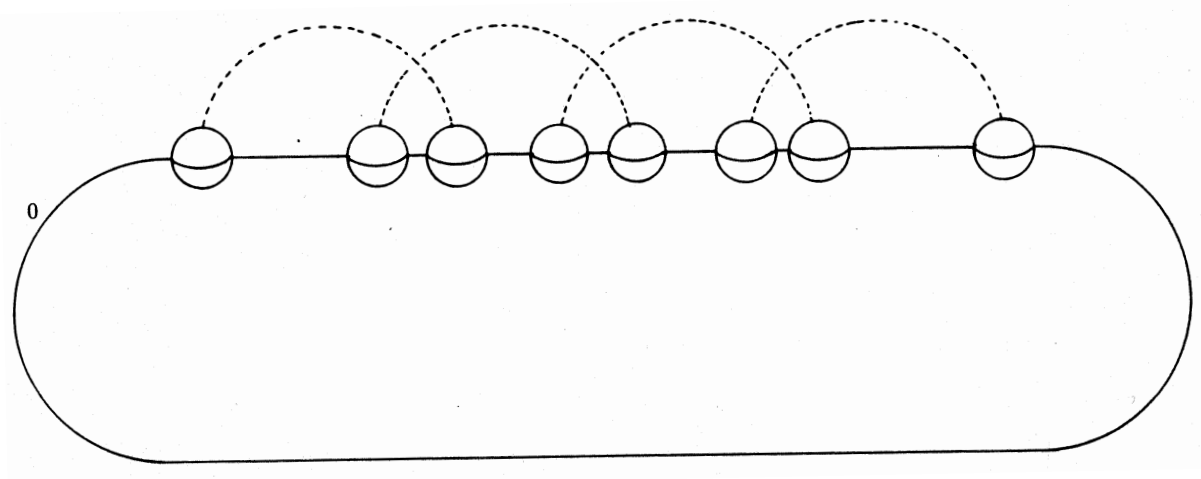

Figure 10.

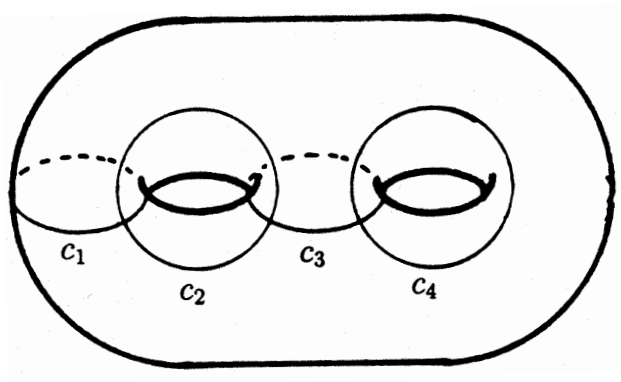

Figure 11. 


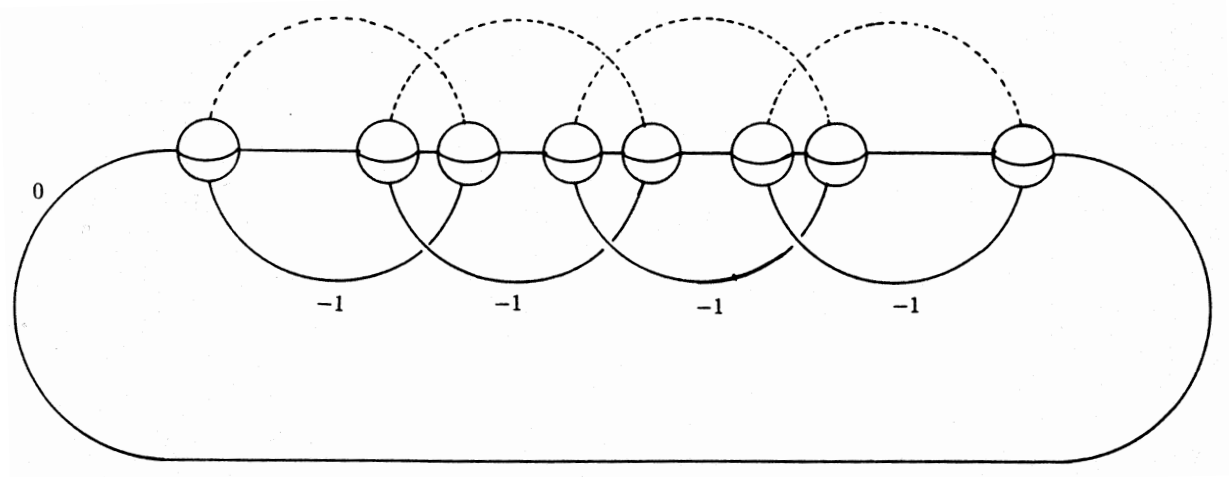

(a)

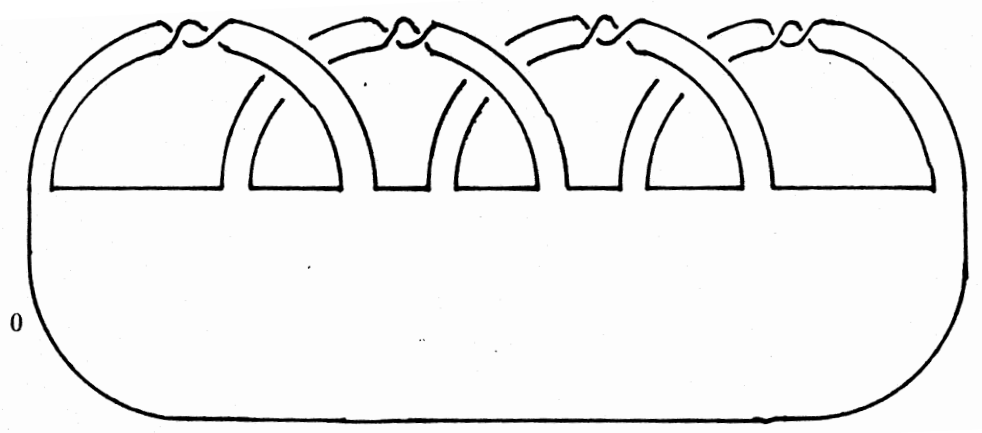

(b)

Figure 12.

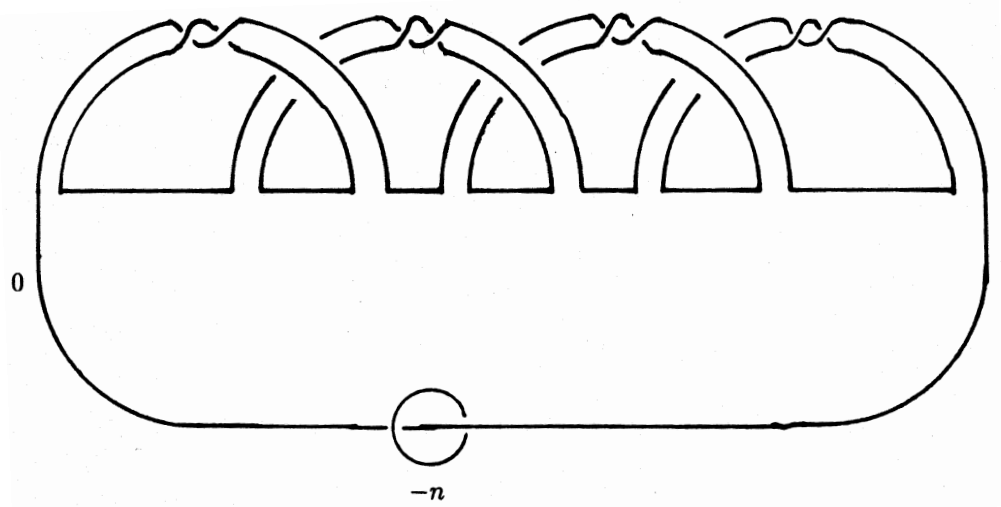

Figure 13. 


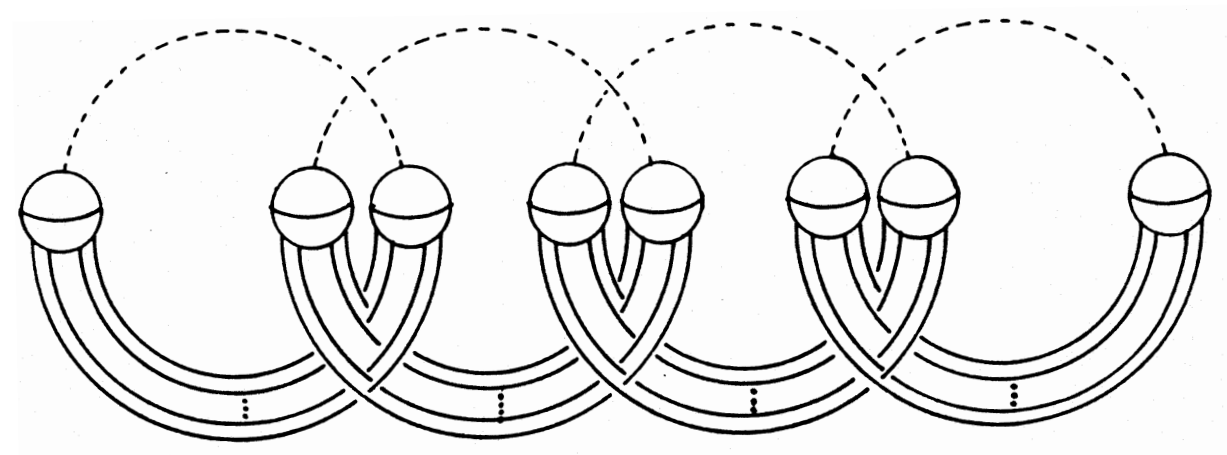

10n-1-1-framed 2-handles per wheel

Figure 14.

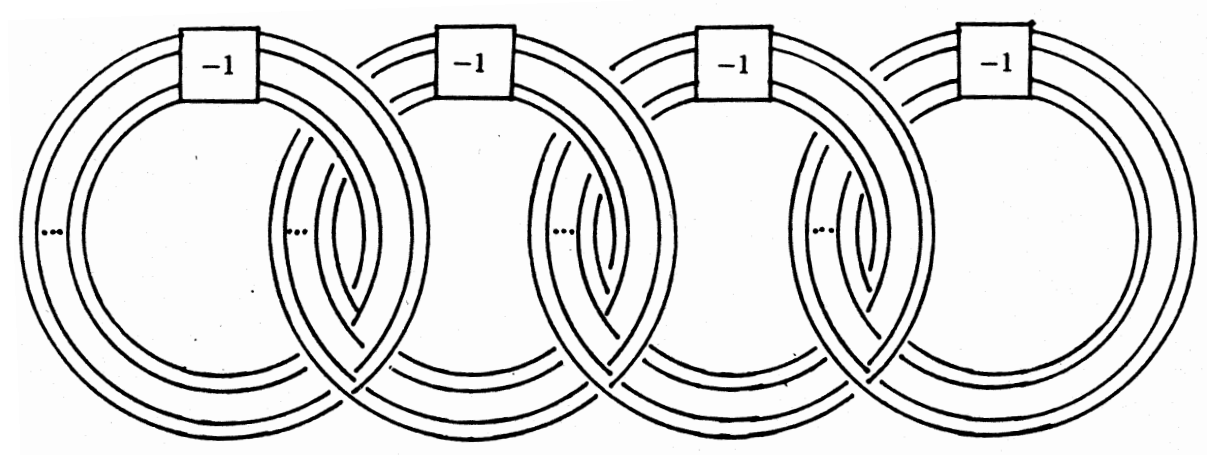

10n-2 -2-framed 2-handles per wheel

Figure 15. 


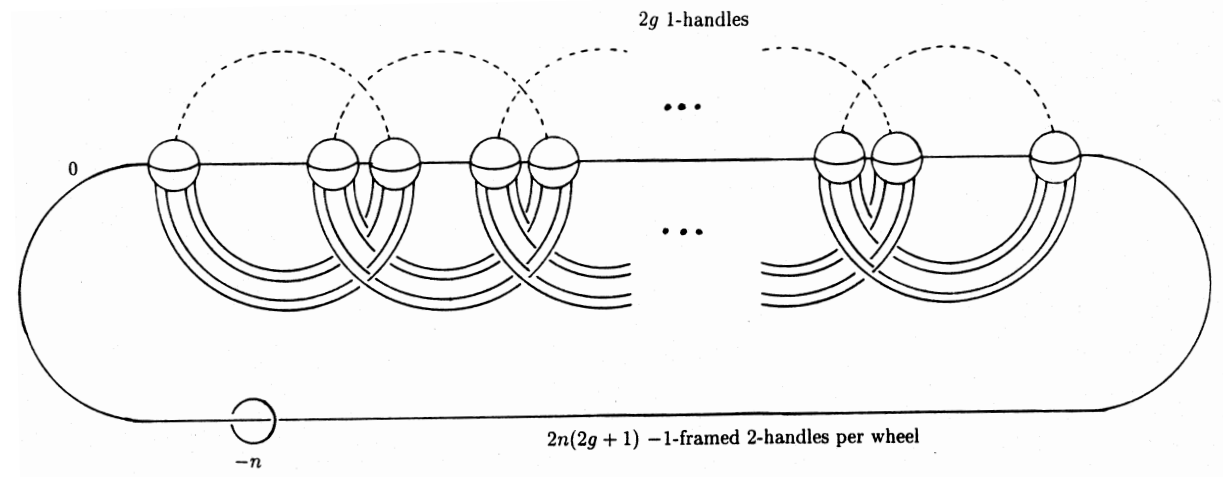

$\cup 2 g$ 3-h, 4-h

Figure 16.

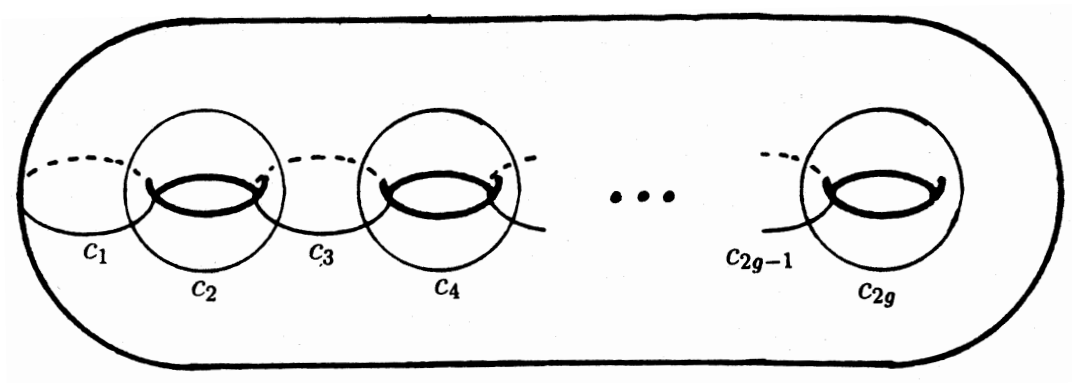

Figure 17.

$2 g$ bands

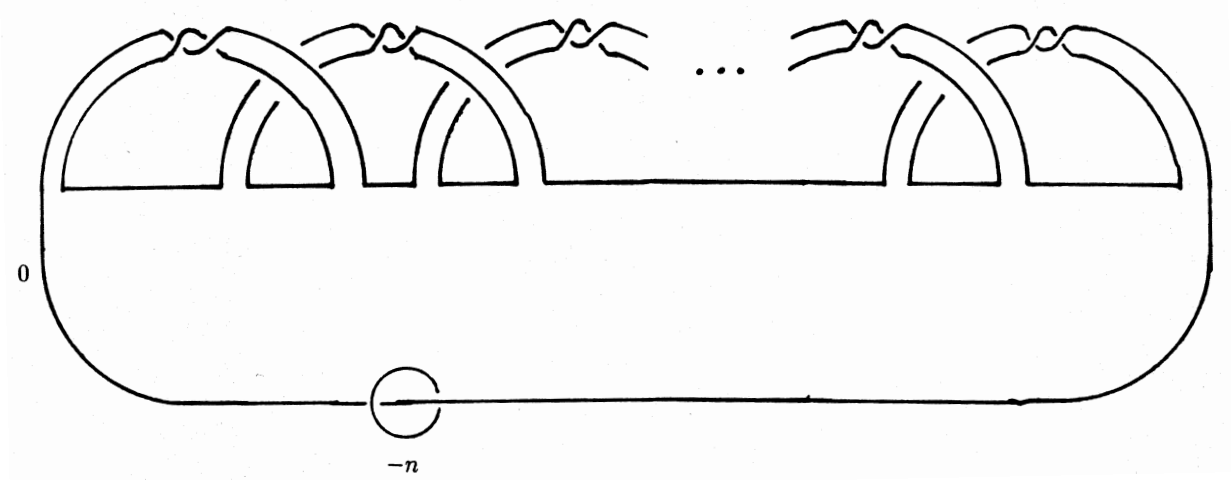

Figure 18. 


\section{References}

[AK] S. Akbulut and R. Kirby, Branched covers of surfaces in 4-manifolds, Math. Ann., 252 (1980), 111-131.

[B] J. Birman, Braids, links, and mapping class groups, Annals of Mathematics Studies, Number 82, Princeton University Press, Princeton, NJ, 1975.

[FS] R. Fintushel and R. Stern, Donaldson invariants of 4-manifolds with simple type, J. Differential Geom., 42 (1995), 577-633.

[Fu] T. Fuller, Diffeomorphism types of genus 2 Lefschetz fibrations, Math. Ann., 311 (1998), 163-176.

[G] R. Gompf, Nuclei of elliptic surfaces, Topology, 30 (1991), 479-511.

[GM] R. Gompf and T. Mrowka, Irreducible 4-manifolds need not be complex, Ann. Math., 138 (1993), 61-111.

[HKK] J. Harer, A. Kas and R. Kirby, Handlebody decompositions of complex surfaces, Mem. Amer. Math. Soc., 62 (1986), number 350.

[K] A. Kas, On the handlebody decomposition associated to a Lefschetz fibration, Pacific J. Math., 89 (1980), 89-104.

[LM] P. Lisca and G. Matić, Tight contact structures and Seiberg-Witten invariants, preprint.

[Mi] J. Milnor, Singular points of complex hypersurfaces, Annals of Mathematics Studies, Number 61, Princeton University Press, Princeton, NJ, 1968.

Received June 10, 1997 and revised November 9, 1997.

UNIVERSITY OF TEXAS

Austin, TX 78712

Institute for Advanced Study

Princeton, NJ 08540 